\title{
A representative driven system to interrogate passive dynamics of an airfoil in the wake of a cylinder
}

\author{
Morgan L. Hooper ${ }^{1 *}$, Beverley J. McKeon ${ }^{1}$ \\ ${ }^{1}$ California Institute of Technology, Graduate Aerospace Laboratories, Pasadena, California U.S.A. \\ *mhooper@caltech.edu
}

\begin{abstract}
Passive motion of an airfoil in the wake of a circular cylinder is compared with driven motion of an airfoil in the same configuration, through simultaneous measurement of both the airfoil dynamics and the surrounding flow field. The passive mounting allows the airfoil to move in the transverse (heaving) direction in response to oncoming forcing, while introducing significant parasitic effects to the dynamics including friction. The driven motion of the airfoil reproduces important characteristics of the imperfect passive motion, validating idealized sinusoidal motion as a model for dynamics of the passive airfoil operating in a more realistic engineering context. Particle Image Velocimetry (PIV) of the driven case is then used to illuminate flow structures contributing to observed power and thrust production in both cases.
\end{abstract}

\section{Introduction}

In 2006, Beal et al. found that a dead fish was able to produce enough thrust to overcome its own drag and 'swim' upstream through interactions with a Von Kármán vortex street shed by an upstream cylinder. It was then demonstrated that a compliantly mounted airfoil placed in a similar wake produced net thrust larger than its net drag, and passively extracted energy from the oncoming flow (Beal et al. (2006)). Thus, dynamics of a passive body reacting to patterns of oncoming vorticity offer insight for applications such as passive drag reduction, efficient vehicle navigation in unsteady flows, or small-scale energy harvesting (for example, see Streitlien et al. (1996), Lefebvre and Jones (2019) or Akaydin et al. (2010)). The behaviour of such systems is complex, combining aerodynamic interactions with oncoming vorticity, non-ideal fluid-structure interactions and added mass effects (for a detailed discussion of added mass, see for example Corkery et al. (2019)). The current work extends the study by Beal et al., combining time-resolved 2D2C Particle lmage Velocimetry (PIV) with direct simultaneous measurement of the forces and motion of an airfoil interacting with the wake of a circular cylinder. The goal of the study is to relate the purely passive dynamics of an airfoil free to move in response to oncoming vorticity to the well-studied case of a driven airfoil in the same configuration, and to evaluate the role particular fluid-structure interactions play in enhancing its power extraction and thrust production potential.

For this purpose, two sets of experiments are compared. One set considers a mechanically mounted airfoil that is allowed to move purely passively in response to oncoming vorticity, but whose dynamics are dominated by realistic parasitic effects such as friction. The other considers an airfoil driven through a prescribed trajectory that captures important features of the passive motion. PIV with a sufficient field of view to interrogate flow structures in the wakes of both the cylinder and the airfoil is performed, while simultaneous motion and force data provide a complete time-resolved picture of fluid-airfoil interactions. We show that an airfoil undergoing purely passive motion induced by the flow, subject to effects of friction and other non-idealities, tends to operate in a regime where the transverse forcing aligns with the velocity while producing mean thrust larger than its mean drag (generating both power and thrust). Through examination of flow structures associated with the motion of the driven airfoil, which exhibits similar performance to the passive case, we are able to identify flow structures which contribute to the observed dynamics. 


\section{Background}

Characterizing the dynamics of an airfoil in vortical flow is an active area of research, particularly for applications in vehicular navigation in an unsteady environment. For example, Lefebvre and Jones (2019) performed force and flow velocity measurements for a stationary airfoil placed downstream of a circular cylinder. For an airfoil at 3 diameters downstream and with the same chord as the cylinder diameter, they observed the formation of a Leading Edge Vortex (LEV) for static, geometric angles of attack ranging from $0-20^{\circ}$ (Lefebvre and Jones (2019)). Consequences of LEV formation and shedding are discussed at length by Eldredge and Jones (2019). Lefebvre and Jones (2019) also demonstrated mean thrust production, even for a stationary airfoil, for most of the cases considered in the study when the geometric angle of attack remained moderate. To connect instantaneous dynamics with the passage of particular structures in the flow, Hufstedler and McKeon (2019) collected time-resolved PIV as well as force measurements of a stationary airfoil encountering an isolated vortical gust. Such studies of stationary airfoils provide a basis to interpret results for more complex studies that allow for either actuated or passive airfoil motion.

Previous work on flapping or otherwise actuated foils in a uniform free stream has largely focused on optimization of pitching and heaving parameters (see for example Xiao and Zhu (2014)) with respect to either thrust production or power extraction. Kinsey and Dumas (2008) proposed that the behaviour of an airfoil undergoing forced harmonic oscillations in both heave and pitch (with a phase difference of $90^{\circ}$ between them) in an oncoming free stream can operate either in a propulsive regime or in a power extraction regime based on the phase relationship between force and velocity. In addition, they showed that the formation of LEVs contributes to improved power extraction by maintaining the alignment between force and velocity over a larger portion of a cycle. If an airfoil is instead allowed to react passively in heave to a driven pitching motion, dynamic parameters of the mounting system also become important. $\mathrm{Su}$ and Breuer (2019) found that the optimal power output for such a semi-passive system was achieved when the driven pitching frequency matched the structural resonance of the mounting system, and the structural damping was tuned to achieve an induced heaving velocity that is in-phase with fluid forces.

A foundational study combining both driven oscillatory motion and interactions with oncoming vorticity is that by Gopalkrishnan et al. (1994). They found that a foil oscillating in both heave and pitch with the same frequency as vortex shedding was able to alter the structure of an oncoming cylinder wake (a drag-type wake) to produce a reverse Von Kármán wake (associated with thrust production) depending on the phase between the motion and oncoming vorticity. They were also able to show that the propulsive efficiency exhibits a strong peak at a phase associated with the emergence of the reverse Von Kármán wake and an overall reduction in combined wake vortex strength. This is reminiscent of the observation from (for example) Kinsey and Dumas (2008) that the switch from propulsion to energy extraction for an oscillating foil manifests as a switch from a thrust wake to a drag wake; however a key distinction between these studies is that for an airfoil interacting with oncoming vorticity, mean power extraction and thrust production may be occurring simultaneously. The present study quantitatively evaluates both the flow structures and forces acting on an airfoil in a similar configuration to that of Gopalkrishnan et al. (1994), to begin disentangling thrust and power-producing interactions.

\section{Experimental Setup}

The airfoil used for both the passive and representative driven experiments is a NACA 0018 airfoil with a chord length of $c=10 \mathrm{~cm}$. Both sets of experiments took place in the NOAH Free-Surface Water Channel facility at the California Institute of Technology. In both cases, the foil was mounted vertically, secured above the tunnel to allow translation in the y-direction (transverse/heave), while its x-direction (streamwise) and z-direction (vertical/spanwise) motion was constrained. A circular cylinder with a diameter of $D=11.5$ $\mathrm{cm}$ was mounted 3 cylinder diameters upstream from the quarter-chord location of the airfoil, creating a Von Kármán wake. The Reynolds number based on cylinder diameter in both cases was approximately 40,000. The basic configuration for both the passive and driven cases is provided in Figure 1 . Note that the position and size of the PIV field of view is approximate, and varied slightly between the two sets of experiments. 


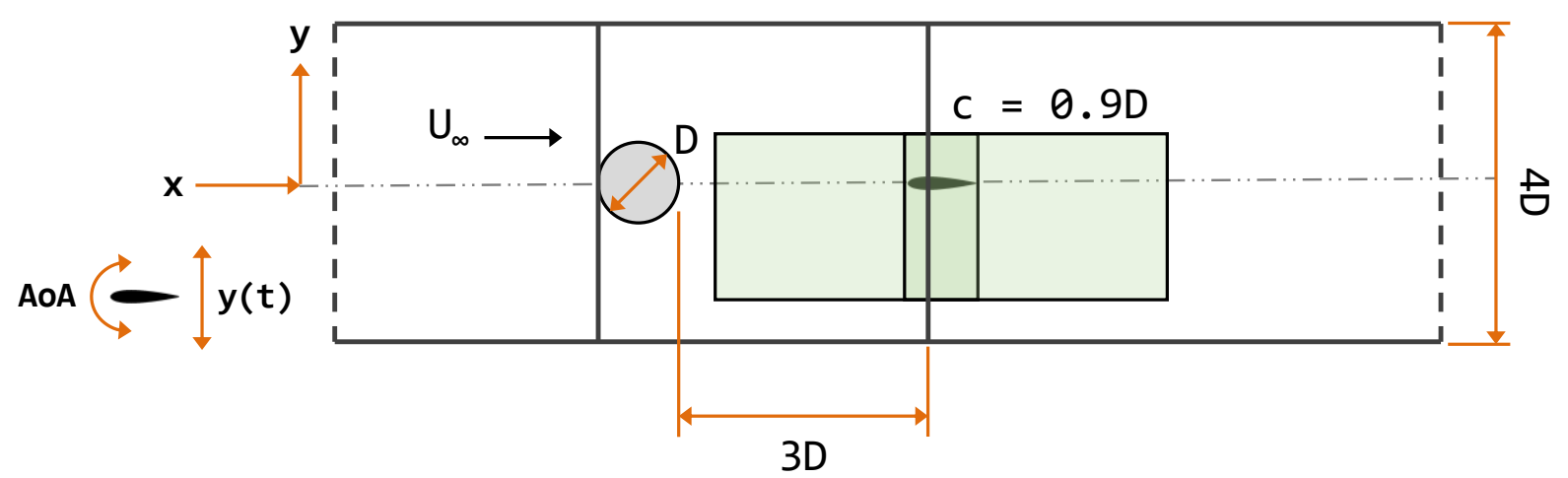

Figure 1: Sketch of water tunnel test section (top view) showing upstream circular cylinder, downstream airfoil. Discussed axes of airfoil motion labelled at left. Green regions indicate PIV field(s) of view.

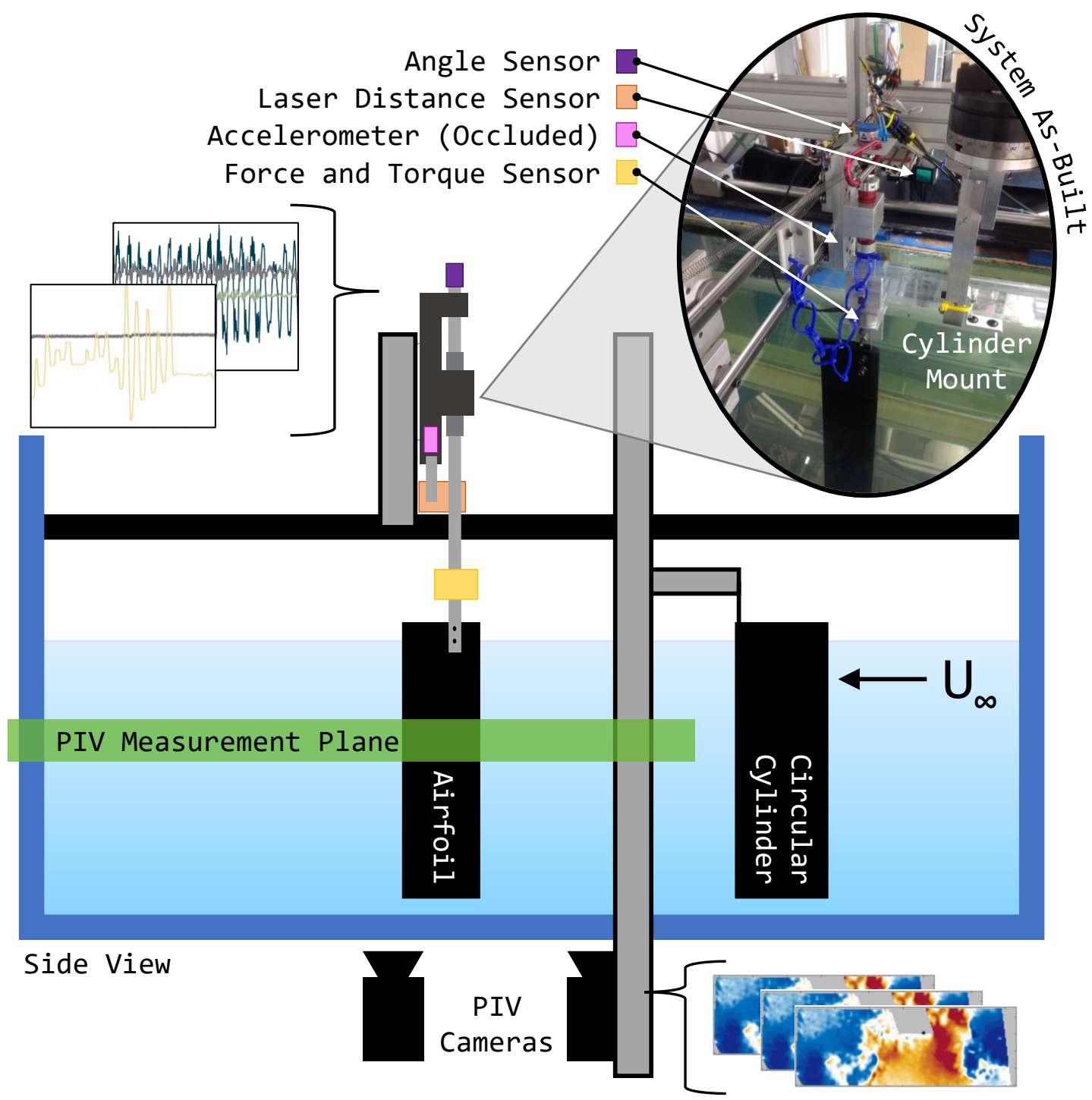

Figure 2: Experiment schematic for the purely passive case. Motion of airfoil and laser sheet extent are into and out of the page. Not to Scale. 


\subsection{Passive Airfoil Configuration}

In the passive configuration, the airfoil was secured to a linear motion cart and allowed to move transversely in response to oncoming free stream vorticity. This mechanical mounting introduced significant friction, which opposed the nominally free motion of the airfoil. The airfoil was mounted to the cart through a rotary bearing on a shaft passing through its quarter-chord location; however, very little change in angle of attack was observed during any individual experimental run, so the airfoil is assumed to be stationary in pitch throughout this analysis (although for each passive run, the stationary pitch angle varied).

The position of the cart was measured using a Keyence LK-G502 laser distance sensor, and the signal was numerically differentiated using a Savitzky-Golay filter to obtain the cart velocity and acceleration as described by Schafer (2011). This method was validated through comparison with the measured acceleration of the cart, obtained using an ADXL 337 accelerometer. The Angle of Attack (AoA) of the airfoil was measured using a Vishay 351 Hall-Effect (HE) rotary encoder. Finally, six-axis forces and torques acting on the airfoil were measured using an ATI Mini40 IP68 force/torque sensor. The laser distance sensor and force/torque sensor included appropriate signal conditioning through dedicated analog-to-digital conversion provided by the manufacturer. The acceleration and angle of attack signals were low-pass filtered prior to data acquisition to ensure compliance with the Nyquist Criterion, and eliminate high-frequency noise. Data were sampled at $25 \mathrm{kHz}$ simultaneously for all sensors. An experimental schematic for the passive airfoil case is given in Figure 2.

\subsection{Driven Airfoil Configuration}

For the driven experiments, the airfoil was mounted to the Captive Trajectory System (CTS), a CyberPhysical Fluid Dynamics (CPFD) system integrated with the NOAH Water Channel. The CTS is able to precisely drive a test object through a prescribed trajectory in the three translational axes, as well as pitch. More details regarding CTS performance were reported by Shamai et al. (2020). Forces and torques on the airfoil were measured using the same force sensor as in the passive experiments; however airfoil position, velocity and force data are available as digital output from the CTS at a rate limited to $200 \mathrm{~Hz}$. The angle of attack of the airfoil was manually adjusted to $0^{\circ}$, and remained fixed at this value for each experiment.

The airfoil was driven through a transverse sinusoidal trajectory with a known frequency and phase relative to the oncoming vortices, of the form

$$
y(t)=A_{p} \sin (2 \pi f t+\psi),
$$

where $y(t)$ is the transverse position as a function of time, and $A_{p}, f$ and $\psi$ were fixed based on data from the passive case.

To determine the appropriate frequency $f$, the transverse force and position from the passive experiments were analyzed. For vortex shedding at a Reynolds Number of 40,000, the value of the Strouhal number,

$$
S t=\frac{f D}{U_{\infty}},
$$

has been shown to remain fixed around a value of 0.2 (for example Lefebvre and Jones (2019), and references therein). This gives an expected dimensional shedding frequency of approximately $0.6 \mathrm{~Hz}$. To confirm that this frequency was dominant in the airfoil dynamics, measured transverse force and position for all passive experiments were windowed into temporal bins varying in length from approximately $5.5 \mathrm{~s}$ to $20.0 \mathrm{~s}$ ( $3.4 T$ to $12.5 T$ ), and linear de-trending was applied to the position signal. Then, the frequency content of each bin was computed using an FFT. In all cases, the windowed signals (with bins of any length) exhibited a strong primary peak in the region of $0.6 \mathrm{~Hz}$; however the position signal sometimes exhibited additional strong peaks at lower frequencies, perhaps due to long-term meander or dynamics induced by the mounting system. To remove this as a source of error in determining dominant oscillatory behaviour due to vortex shedding, only the transverse force signals were used to compute the oscillation frequency for the driven experiments. The peak locations in all transverse force signal bins were averaged resulting in the selection of $0.6226 \mathrm{~Hz}$, which gives a Strouhal number of 0.22 .

To determine the appropriate phase offset between the measured force and the airfoil motion (a proxy for the phase between the vortex shedding and the airfoil motion), the difference in phase angle computed 
from the FFT at the dominant forcing frequency in each bin between the force and motion was averaged. Although this phase difference varied widely especially for shorter window lengths, the mean value was determined to be quite close to $\pi / 2$. This value confirms a visual trend identified in the passive airfoil data, as well as enforcing the condition that the airfoil's velocity is in-phase with the forcing. This is intuitively satisfying as it enforces that the rate of work done on the airfoil by the surrounding flow,

$$
P(t)=F_{y}(t) \dot{y}(t)
$$

is always positive. Since there is no power input available for the airfoil to do mean work on the flow, this is a physically meaningful constraint; thus, for the driven sinusoidal motions discussed in the following sections, the phase $\psi$ for the motion is set such that velocity and transverse force are (approximately) in-phase.

Finally, the appropriate amplitude for the driven sinusoidal motion was determined through analogy with the time-mean kinetic energy of the passive airfoil. Considering a pure sinusoid,

$$
\bar{E} \propto \lim _{T \rightarrow \infty} \frac{1}{T} \int_{0}^{T}\left[A_{v} \sin (\omega t)\right]^{2} d t=\frac{A_{v}^{2}}{2} .
$$

Then, the mean kinetic energy of a signal that is not perfectly periodic can be matched to an equivalent value for a pure sinusoid. We consider:

$$
\begin{gathered}
\bar{E}=\frac{A_{v}^{2}}{2} \\
A_{v}=\sqrt{2 \bar{E}}=\omega A_{p} \\
A_{p}=\frac{\sqrt{2 \bar{E}}}{\omega}
\end{gathered}
$$

where $A_{v}$ is the amplitude of the velocity, and $A_{p}$ is the amplitude of the position for a pure sinusoid. By calculating $\bar{E}$ as the mean kinetic energy over all runs (ie, by summing square velocity at each recorded time step for all experimental runs for the passive case then dividing by total time steps), then applying Equation 7, we can estimate the appropriate amplitude for our synthetic position signal as $A_{p}=5.1 \mathrm{~mm}$, or approximately $5 \%$ of the airfoil chord.

In summary, for the driven case reported in this paper, the airfoil was driven through a prescribed sinusoidal trajectory given by the following equation (for position normalized by airfoil chord):

$$
y^{*}(t)=0.05 \sin (2 \pi(0.6226) t+\psi)
$$

where $\psi$ was fixed for each individual run to ensure that the velocity was in-phase with the force acting on the airfoil due to vortex shedding.

\subsection{PIV System}

For both the passive and driven cases discussed above, 2D2C PIV was performed simultaneously with measurements of airfoil dynamics. PIV images were captured at a rate of $800 \mathrm{~Hz}$ using two Phantom Miro Lab 320 cameras with a pixel resolution of $1920 \times 1200$ px. The two fields of view partially overlapped to form a single, continuous region of interest (see Figure 11). Both cameras were placed directly beneath the tunnel, perpendicular to the transparent tunnel bottom, and equipped with $35 \mathrm{~mm}$ Nikkor lenses. Neutrally buoyant tracer particles are illuminated by a Photonics DM20-527(nm) YLF laser in single-pulse mode, which was expanded through a cylindrical lens to form a sheet. The laser sheet entered the tunnel through a side wall parallel to the tunnel bottom.

PIV image acquisition and processing was completed using commercial software (LaVision DaVis, version 10.1.2). Raw images were first averaged to compute a background image, which was subtracted from each frame prior to processing. In addition, the outline and shadow created by the airfoil was masked out based on its measured position in each frame. Prepared images are then processed sequentially to produce vector fields. A multi-pass correlation algorithm was used, where an initial pass using a 64x64 pixel square 

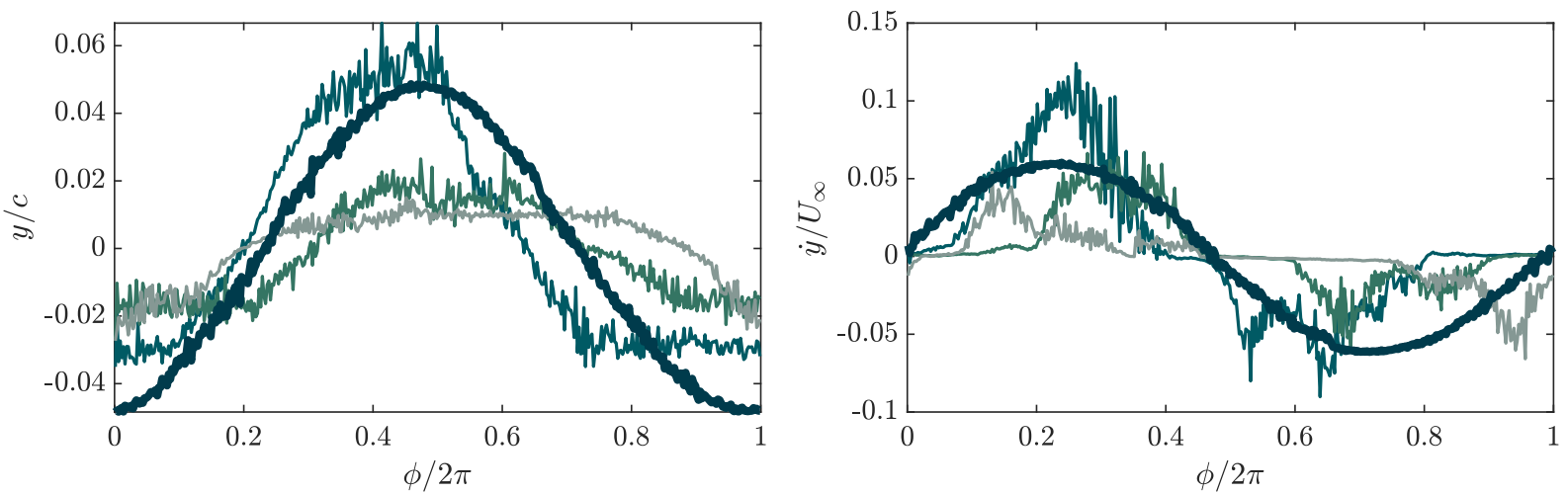

Figure 3: Airfoil phase-averaged position (left) and velocity (right) over one vortex shedding cycle. Colours correspond to the following cases: DR; PA10; - PA11; PA12.

window was subsequently reduced to yield a final circular window size of $16 \times 16$ pixels, through three additional passes. Each pass included a 50\% overlap between windows, and outliers were removed between each pass based on a minimum correlation value threshold of 0.4 , as well as median filtering. Obtained vector fields were then post-processed using an additional median filter to remove outliers, as well as a $3 \times 3$ smoothing filter to reduce noise in the final fields. The resulting size of the field of view was $0.65 \times 0.24 \mathrm{~m}$ $(5.7 \times 2.1 D)$ in the driven case, and $0.67 \times 0.24 \mathrm{~m}(5.8 \times 2.1 D)$ in the passive cases.

\section{Phase Reference Determination for Passive and Driven Cases}

To define a global phase reference for both the driven and passive experiments, a Proper Orthogonal Decomposition (POD) of the oncoming flow was computed. Flow behind a circular cylinder exhibits Von Kármán vortex shedding, which has a relatively compact representation in a basis formed by its POD modes. This can be exploited to create a phase reference for vortex shedding based on direct observations of the flow field, as demonstrated by Lefebvre and Jones (2019) and van Oudheusden et al. (2005). This is particularly useful for determining a quantitative phase reference in the passive case, where the motion and measured forcing is not perfectly periodic. It also allows for a more objective comparison between the driven and passive cases with respect to flow behaviour, which remains the same for both.

Thus, in the following sections the global phase of the cylinder-airfoil system is computed based on a proper orthogonal decomposition of the flow field upstream of the location of the airfoil (before flow around the airfoil disrupts the pattern of vortex shedding). First, the first two POD modes for this reduced flow field are calculated, and their projection coefficients at each PIV time step are determined. Then, the phase angle is calculated by considering (as in van Oudheusden et al. (2005))

$$
\phi=\operatorname{atan}\left(\frac{A_{2} \sqrt{\sigma_{1}}}{A_{1} \sqrt{\sigma_{2}}}\right),
$$

where $A_{1}, A_{2}$ are the first and second POD mode coefficients, and $\sigma_{1}, \sigma_{2}$ are the associated singular values.

\section{Comparison between Driven and Passive Airfoil Dynamics}

Using the global phase reference given by Equation 9 , position, velocity, lift, thrust and power data from the passive and driven cases are phase-averaged to reveal underlying trends associated with vortex shedding. The data are binned into 400 bins of equal width corresponding to 0.0157 radians or 0.0040 seconds, based on the estimated mean shedding period of $T=1.6062 \mathrm{~s}$. Figure 3 shows the phase-averaged position and velocity of the airfoil over one shedding cycle for both the driven case (case DR, the bolded line), and three different passive cases (cases PA10, PA11 and PA12), each with a different static angle of attack and mean tunnel position. As these factors affect airfoil performance, the passive cases were not phase-averaged 
together and instead are reported separately as thin lines in Figure 3. A moving average was removed from the position signals for the passive case prior to phase averaging, to more clearly highlight the cyclic character of the airfoil motion and remove any mean position offset. In the driven case, phase averaging was only performed for periods when the velocity and measured forcing were in-phase to within 5\% of $T$, to enforce this alignment for the phase-averaged data despite slight meander in fluid forcing frequency.

The driven sinusoidal trajectory appears to capture the phase-averaged passive airfoil motion qualitatively well as evidenced by Figure 3, although the passive cases exhibit some spread in the location of the velocity peaks. This is likely due to the variation in test conditions, as well as inherent cycle-to-cycle variability induced by non-ideal and nonlinear effects such as friction. Frictional effects also induce the stick-slip motion observed in cases PA10-12, where the airfoil experiences brief periods of non-zero velocity surrounded by periods of inactivity rather than a smooth sinusoidal velocity variation as in case DR. In addition, phase averages for each passive case (PA10-12) are based on total time periods of 2.9T, corresponding to a single PIV run each. By contrast, operating conditions were held constant in the driven experiments by design, allowing many individual runs to be averaged together in case DR. Therefore higher variability in the phase-averaged results for the passive cases (PA10-12) is expected.

Figure 4 shows the phase-averaged thrust, lift and power coefficients for the driven and passive cases discussed above, where the coefficients are defined in the conventional manner as

$$
C_{T}=\frac{T}{\frac{1}{2} \rho U_{\infty}^{2} s c} \quad C_{L}=\frac{L}{\frac{1}{2} \rho U_{\infty}^{2} s c} \quad C_{P}=\frac{P}{\frac{1}{2} \rho U_{\infty}^{3} s c},
$$

where $s$ is the span of the airfoil submerged in the tunnel. The first panel shows the phase-averaged velocity reproduced from Figure 3 for reference. Again we see relatively good qualitative agreement between the behaviour in both the driven case and the passive cases. In all cases, we observe a roughly sinusoidal trend in $C_{L}$ induced by the passage of shed vorticity from the upstream cylinder; in addition we see that for all cases the phase-averaged velocity of the airfoil is roughly in-phase with this forcing (an enforced condition for case DR). Although the passive cases seem to generally confer a maximum $C_{L}$ benefit (larger amplitude variation in $C_{L}$ ), the phase-averaged power coefficient varies widely between passive runs. This
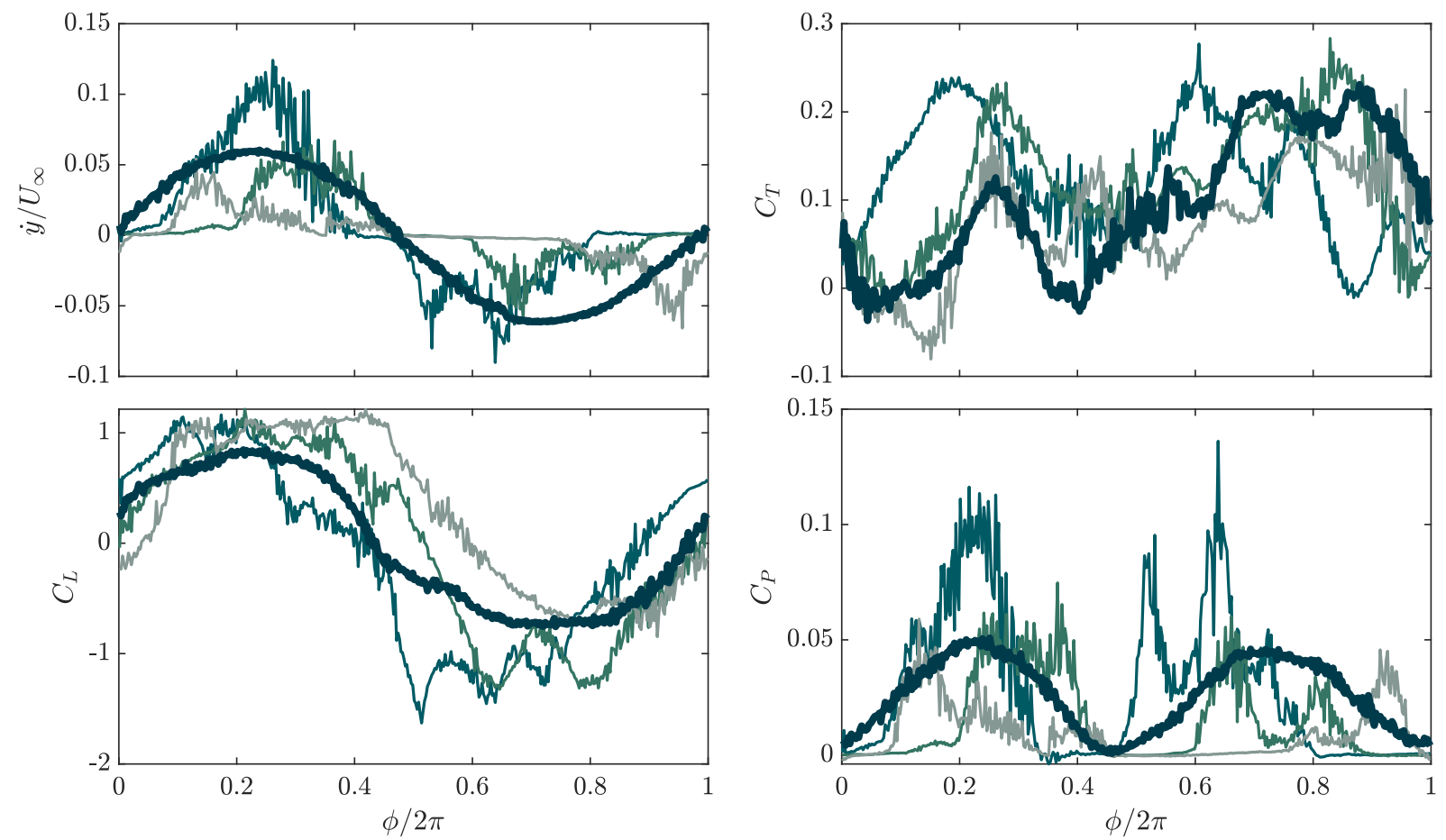

Figure 4: Phase-averaged velocity (top left), thrust coefficient (top right), lift coefficient (bottom left) and power coefficient (bottom right) for passive and driven cases. DR; - PA10; $=$ PA11; - PA12. 
effect appears to be caused by longer periods of inactivity in some of the passive cases, caused by the action of friction opposing the startup of airfoil motion (evidenced by extended periods where $\dot{y}=0$, especially for PA11 and PA12). However, when the airfoil does move, the power production in the passive case appears to exceed that of the driven case (at least in one recorded case, PA10). The action of friction in the transverse direction does not appear detrimental to thrust production: positive thrust production (in addition to positive power, or power extraction) is experienced at a similar magnitude in both the driven and passive cases.

To investigate the flow features responsible for simultaneous power extraction and thrust production in these systems, phase-averaged velocity fields are discussed in the following section. Based on the preceding discussion, we examine velocity fields for the driven case (DR) only, as the longer available time periods for averaging provide a clearer picture of the cyclic flow features of interest. The highly variable nature of the airfoil behaviour in the passive case presents a challenge in creation of a representative phase average velocity field in the region of the airfoil, and is therefore deferred for future exploration; however the similarity in the dynamics observed between the passive and driven cases above validates the use of the driven case as a model at least for the large-scale cyclic behaviour in the more complex passive case(s).

\section{Observed Flow Structures and Influence on Dynamics}

Figures 5 and 6 provide phase-average snapshots of the flow field around the airfoil at three different vortex shedding phases, as indicated by the dashed lines in the left-hand panels. Vortex shedding phase advances from top to bottom in the images on the right for each figure. In Figure 5, the transverse (y-direction) velocity is provided. In Figure 6, values of the $\Gamma_{2}$ criterion (a proxy for vorticity, as described by Graftieaux et al. (2001)) for the same phase locations are provided.

The top-right panel in each of Figures 5 and 6 shows the flow at the moment corresponding to the first of two power production peaks observed in the cycle. We see that the airfoil is passing through its neutral position behind the cylinder, and experiencing its maximum upwards velocity. It is centered in a region of upwash generated by upstream vortex shedding, as shown in Figure 5, and flow over the top of the airfoil is relatively well-ordered leading to a local maximum in the lift force. It is interesting to note that the peak in the thrust lags slightly behind the peaks in lift and power: this could indicate a stronger dependence of the thrust production on the location of the upstream vortices (global flow conditions), rather than the local conditions surrounding the airfoil itself. For example, in Figure 6 in the top right panel we see that the airfoil is located downstream and above a counter-clockwise rotating vortex, but upstream and below a clockwise rotating vortex. Both of these structures contribute to upstream forcing on the airfoil (though we emphasize that they do not create a region of reverse flow near the airfoil).

The middle-right panel in each of Figures 5 and 6 shows a moment midway between the peak and trough in the observed power production, and at the beginning of a trough in the thrust. We see strong evidence of the growth of an LEV attached to the airfoil, especially in Figure 6, as well as flow separation as indicated by the region of negative flow close to the airfoil's surface in Figure 5, despite its positive velocity. We see that the $C_{L}$ value at this moment remains high, with a sharper downwards slope at phases just beyond; as discussed by Kinsey and Dumas (2008), the growth of an LEV may be helping to keep the lift high for the high-velocity portion of the cycle, resulting in augmented power production. By contrast, the oncoming counter-clockwise rotating vortex has encountered the airfoil, thereby losing some coherence: this could be contributing to the loss of thrust at this phase.

The bottom-right panel in each of Figures 5 and 6 shows a moment of minimum power production, which coincides with a maximum in airfoil position and therefore a zero-point in the velocity. Since $P=F_{y}(t) \dot{y}(t)$, this velocity zero crossing is largely responsible for the dip in power output. At this moment, we see that the airfoil is perched between regions of upwash and downwash (engulfed in a counterclockwise-rotating vortex), and the flow over the airfoil's surface is separated and highly disordered with regions of downwards flow especially evident over the trailing edge. In addition, the fast flow past the leading edge has been attenuated by the advancement of a region of downwash. These factors contribute to the small lift values experienced at this point in the cycle. Interestingly, the thrust coefficient has passed its local minimum and has started to recover. This provides further evidence that thrust production behaviour may depend more strongly on global conditions, such as the advance of the new oncoming clockwise-rotating vortex upstream of the airfoil. 

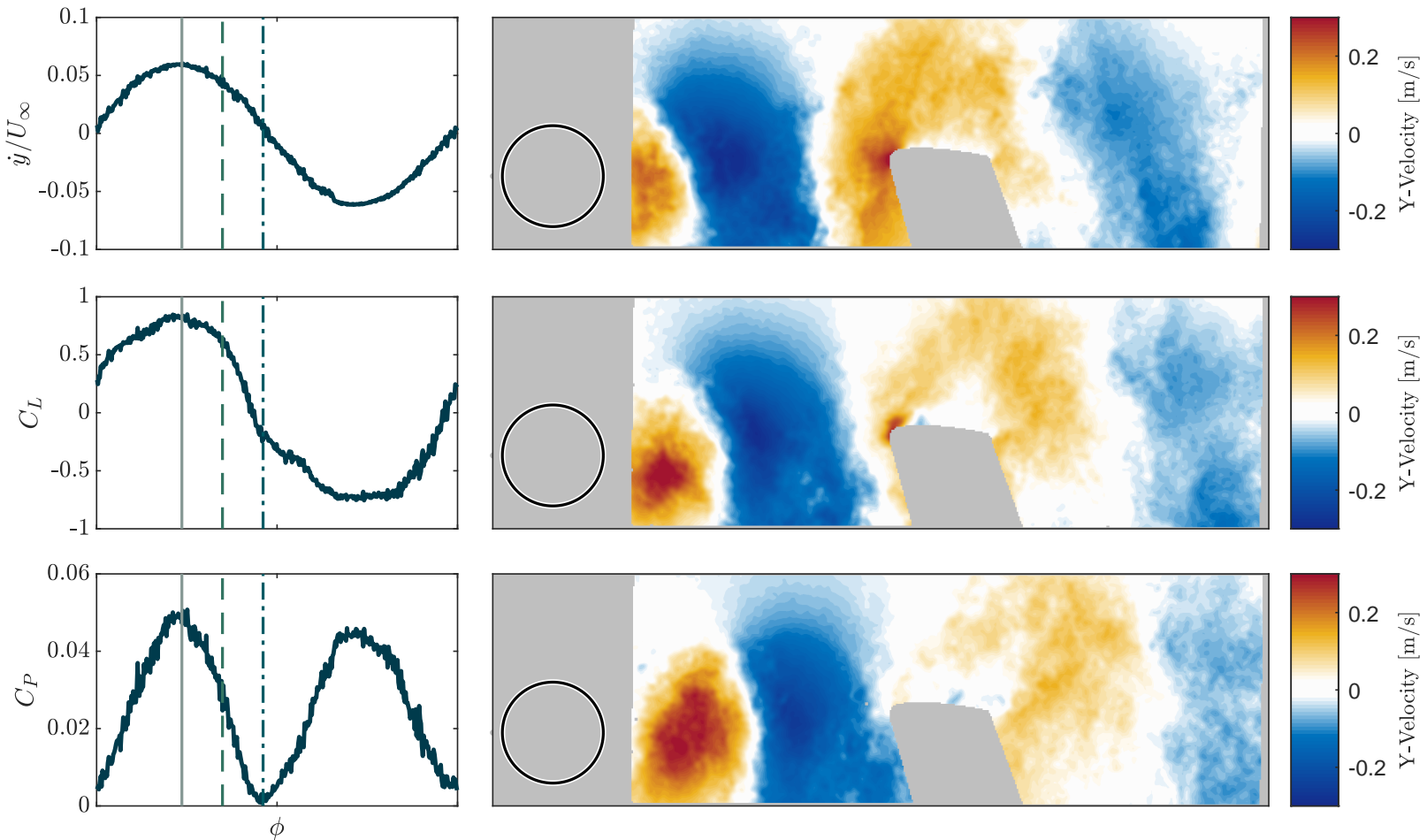

Figure 5: Snapshots of phase-averaged transverse velocity at indicated points in the vortex shedding cycle. Left column reproduces data for case DR from Figure 4. Phase-averaged Velocity (top), Lift Coefficient (middle) and Power Coefficient (bottom). Left-hand figures also indicate the phase of each snapshot shown on right: $=$ Top Panel; = - Middle Panel; - י Bottom Panel.
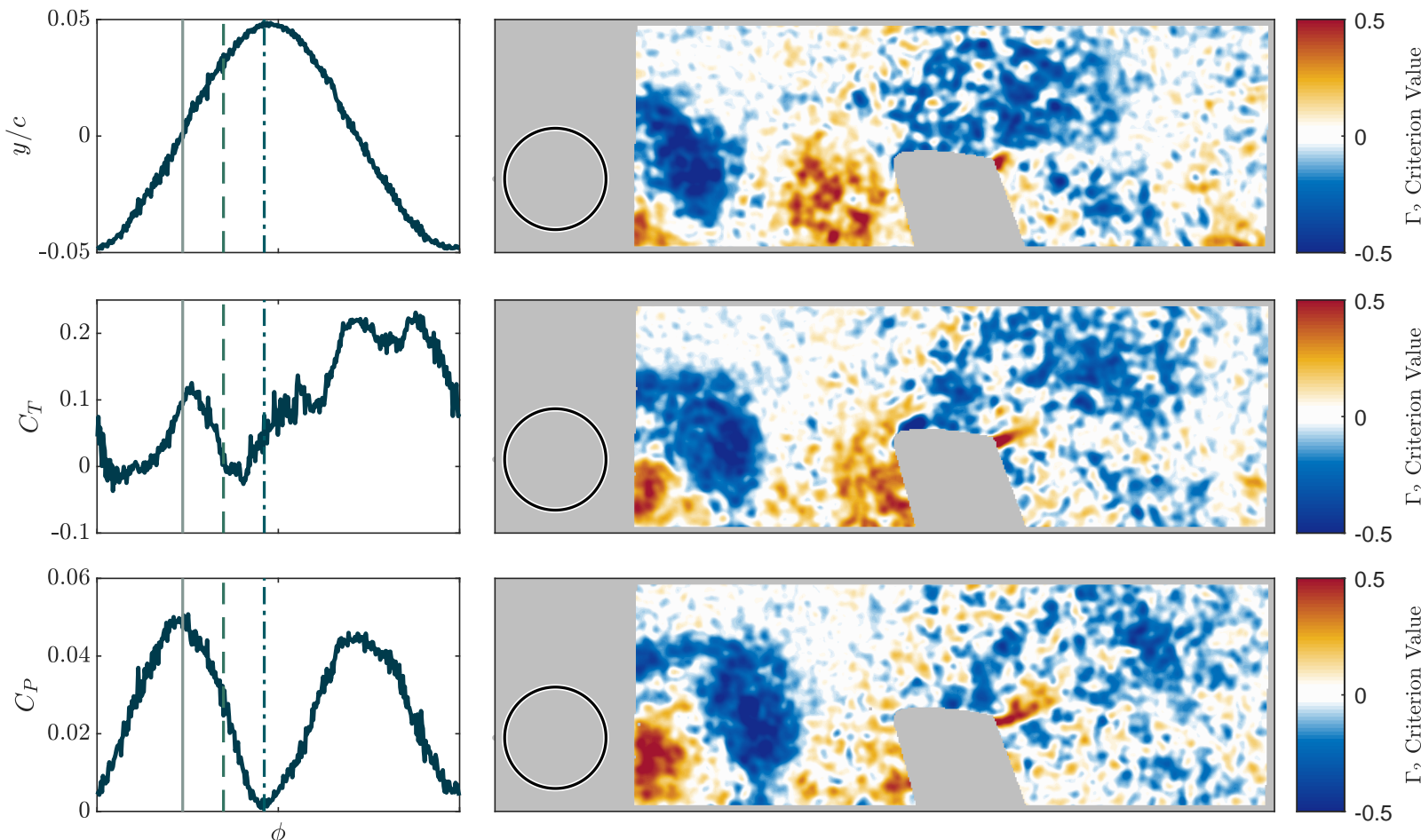

Figure 6: Snapshots of phase-averaged $\Gamma_{2}$ criterion values at indicated points in the vortex shedding cycle. Left column reproduces data for case DR from Figures 3 and 4; Phase-averaged Position (top), Thrust Coefficient (middle) and Power Coefficient (bottom). Left-hand figures also indicate the phase of each snapshot shown on right: $=$ Top Panel; $=$ - Middle Panel; - - Bottom Panel. 


\section{Conclusions}

Experiments were performed to interrogate the behaviour of an airfoil in the wake of a circular cylinder, both when the airfoil was free to interact with oncoming vorticity, and when it was driven through an idealized sinusoidal trajectory. Time-resolved PIV coupled with measurements of airfoil dynamics allowed the creation of a phase-averaged picture of the airfoil behaviour in both cases, and it was shown that the idealized driven trajectory captures salient features of the more variable passive motion. Thus, phase-averaged velocity and $\Gamma_{2}$ fields for the driven case were analyzed to determine dominant flow structures linked to the behaviour of the airfoil. Simultaneous production of thrust and power are linked strongly to cyclic interactions with both regions of upwash and downwash as well as coherent vortices shed by the upstream cylinder.

\section{Acknowledgements}

The support of the US Army Research Office under grant number W911NF-17-1-0306, as well as that from the Natural Sciences and Engineering Research Council of Canada (NSERC) is gratefully acknowledged.

\section{References}

Akaydin HD, Elvin N, and Andreopoulos Y (2010) Wake of a cylinder: a paradigm for energy harvesting with piezoelectric materials. Experiments in Fluids 49:291-304

Beal DN, Hover FS, Triantafyllou MS, Liao JC, and Lauder GV (2006) Passive propulsion in vortex wakes. Journal of Fluid Mechanics 549:385-402

Corkery SJ, Babinsky H, and Graham WR (2019) Quantification of added-mass effects using particle image velocimetry data for a translating and rotating flat plate. Journal of Fluid Mechanics 870:492-518

Eldredge JD and Jones AR (2019) Leading-Edge Vortices: Mechanics and Modelling. Annual Review of Fluid Mechanics 51:75-104

Gopalkrishnan R, Triantafyllou MS, Triantafyllou GS, and Barrett D (1994) Active vorticity control in a shear flow using a flapping foil. Journal of Fluid Mechanics 274:1-21

Graftieaux L, Michard M, and Grosjean N (2001) Combining PIV, POD, and vortex identification algorithms for the study of unsteady turbulent swirling flows. Measurement Science and Technology 12:1422-1429

Hufstedler EAL and McKeon BJ (2019) Vortical Gusts: Experimental Generation and Interaction with Wing. AIAA Journal 57:921-931

Kinsey T and Dumas G (2008) Parametric Study of an Oscillating Airfoil in a Power-Extraction Regime. AIAA Journal 46:1318-1330

Lefebvre JN and Jones AR (2019) Experimental Investigation of Airfoil Performance in the Wake of a Circular Cylinder. AIAA Journal 57:2808-2818

Schafer RW (2011) What Is a Savitzky-Golay Filter?. IEEE Signal Processing Magazine pages 111-117. Date of Publication: 15 June, 2011.

Shamai M, Dawson STM, Mezić I, and McKeon BJ (2020) From unsteady to quasi-steady dynamics in the streamwise-oscillating cylinder wake. arXiv 2007.05635v1[physics.flu-dyn]. Phys. Rev. Fluids, to appear.

Streitlien K, Triantafyllou GS, and Triantafyllou MS (1996) Efficient Foil Propulsion Through Vortex Control. AIAA Journal 34:2315-2319

Su Y and Breuer K (2019) Resonant response and optimal energy harvesting of an elastically mounted pitching and heaving hydrofoil. Physical Review Fluids 4:064701-1:18

van Oudheusden BW, Scarano F, van Hinsberg NP, and Watt DW (2005) Phase-resolved characterization of vortex shedding in the near wake of a square-section cylinder at incidence. Experiments in Fluids 39:86-98

Xiao Q and Zhu Q (2014) A review on flow energy harvesters based on flapping foils. Journal of Fluids and Structures 46:174-191 